\title{
BOMBILLAS DE BAJO CONSUMO
}

\section{ENERGY EFFICIENT LIGHT BULBS}

Julio Gutiérrez Muñoz: Universidad de Alcalá de Henares. Madrid (España) julio.gutierrez@uah.es

\section{CURRÍCULUM VITAE}

Catedrático de Física Atómica, Molecular y Nuclear de la Escuela Politécnica Superior de la Universidad de Alcalá de Henares (Madrid). En la actualidad imparte clases de la asignatura de Física incluida en la titulación de Grado en Química de dicha Universidad. Autor de numerosos artículos científicos. Miembro fundador de GRUA (Grupo de Reflexión de la Universidad de Alcalá de Henares). Vicerrector de la Universidad de Alcalá de Henares. Director de la Revista Vivat Academia.

\section{RESUMEN}

BFC son las iniciales de Bombilla Fluorescente Compacta. Estas lámparas son vulgarmente llamadas bombillas de bajo consumo. La única diferencia real entre un tubo fluorescente clásico y una BFC es su tamaño, lo cual hace más puntual el foco emisor de luz, disminuyendo su eficacia en iluminación. El primer dispositivo antecesor de la lámpara fluorescente fue el tubo de Geissler, que producía una luz azulada gracias a un gas noble. La principal ventaja de una BFC frente a una bombilla de incandescencia es su eficiencia energética. Aproximadamente, se calcula un ahorro de un 25\% para un nivel de iluminación similar. La principal desventaja es 
que la luminosidad de la lámpara depende fundamentalmente de la superficie emisora, es decir de la superficie del cilindro iluminado.

\title{
PALABRAS CLAVE
}

Bombilla fluorescente compacta - Bajo consumo - Tubo fluorescente - Iluminación

\begin{abstract}
BFC stands for Compact Fluorescent Lamp. These lamps are commonly called light bulbs. The only real difference between a traditional fluorescent tube and a BFC is its size, which makes it point the light emitting source, decreasing its efficiency in lighting. The first device ancestor of the fluorescent lamp was the Geissler tube, producing a bluish light thanks to a noble gas. The main advantage of BFC versus an incandescent lamp is energy efficient. Roughly estimated a savings of $25 \%$ for a similar light. The main disadvantage is that the brightness of the lamp depends crucially on the emitting surface, so the cylinder surface illuminated.
\end{abstract}

\section{KEY WORDS}

Compact fluorescent bulb - Energy - Fluorescent tubes - Lighting

\section{ÍNDICE}

1. Introducción

2. Un poco de historia

3. Principio de funcionamiento

4. Ventajas no tan ventajosas

5. Desventajas 


\section{TEXTO}

\section{Introducción}

La única diferencia real entre un tubo fluorescente clásico, mostrado por primera vez al público en la Feria Mundial de New York del año 1939, y una BFC es su tamaño, reducido en esta última por el simple método de enrollar el tubo, lo cual hace más puntual el foco emisor de luz, disminuyendo su eficacia en iluminación. Desde esa fecha, no ha cambiado el principio de su funcionamiento, únicamente se han introducido las mejoras correspondientes a los avances tecnológicos, permitiendo una reducción de su precio y dimensiones, extendiendo su uso a los hogares. No obstante, la necesidad de utilizar elementos adicionales para su encendido y funcionamiento continuo, hace que el volumen de los soportes sea considerablemente grande y antiestético. Aplicado a las BFC, ello supone un casquillo demasiado voluminoso, imposibilitando en la práctica su utilización en los modelos actuales de lámparas más o menos artísticas de una casa particular.

\section{Un poco de historia}

El primer dispositivo considerado como antecesor de la lámpara fluorescente, llamado "Tubo de Geissler" fue ideado por el físico alemán Heinrich Geissler, en 1856. Consistía en un tubo lleno de un gas noble (neón o argón) que se excitaba mediante una bobina de inducción y producía una luz azulada. (El lector recordará la luminosidad producida por un tubo fluorescente moderno en las proximidades del campo magnético variable producido por las líneas de alta tensión).

Nikola Tesla, colaborador de Tomas Alva Edison, también mostró dispositivos fluorescentes en la Feria Mundial de 1893, en competencia con la bombilla de incandescencia ideada por su antiguo jefe. Asimismo, un antiguo empleado de 
Edison, Daniel McFarlane Moore, desarrolló, en 1894, una lámpara comercial de descarga gaseosa (contenía nitrógeno y dióxido de carbono), la "Lámpara Moore", también en competencia con las lámparas que salían de la factoría Edison. Sin embargo, tuvo relativo poco éxito, debido, sobre todo, a sus dificultades de instalación, mantenimiento y reparación. Estas lámparas producían una luz blanco rosada y fueron instaladas, por vez primera, en unos almacenes de la ciudad de Newark (USA).

La lámpara más parecida en diseño a los actuales tubos fluorescentes fue ideada por Peter Cooper Hewitt, en 1901, utilizando como gas vapor de mercurio. Estos dispositivos, de mayor eficiencia energética que las lámparas de incandescencia, producían, sin embargo, una luz azulado verdosa inadecuada para la iluminación.

En 1926, Edmund Germer, Friedrich Meyer y Hans Spanner aumentaron la presión del gas dentro del tubo y recubrieron éste internamente con un polvo fluorescente. De esta forma, la radiación ultravioleta emitida por el plasma de mercurio era absorbida y posteriormente reemitida por el recubrimiento en forma de luz visible casi blanca (el mismo diseño actual). Tras patentar su invento en 1927, vendieron los derechos a la empresa General Electric, quien lo comercializó en 1938, gracias a las mejoras introducidas, consistentes en utilizar los actuales tubos rectos, encendidos por precalentamiento, bajo la dirección de George E. Inman..

Hasta 1980, año en que Philips lanzó la primera lámpara ahorradora compacta, los tubos fluorescentes habían cambiado poco -o nada- en diseño y tecnología. Esa lámpara, denominada Ambiance, se parecía bastante a las bombillas incandescentes normales.

En los últimos años, las BFC se han hecho cada vez más pequeñas, ligeras y eficaces. 


\section{Principio de funcionamiento}

Todos hemos oído hablar de los complementos de un tubo fluorescente: el cebador y la reactancia. Veamos a continuación el porqué de su necesidad.

Una lámpara fluorescente está formada por un tubo de vidrio, revestido interiormente con una sustancia que contiene un compuesto de fósforo y otros elementos que emiten luz al recibir una radiación de frecuencia ultravioleta. El tubo contiene una pequeña cantidad de vapor de mercurio y un gas inerte, habitualmente argón, sometidos a una presión ligeramente inferior a la presión atmosférica, lo cual hace que el mercurio, líquido a temperatura ambiente, se vaporice.

Para que el átomo de mercurio emita luz primero debe "excitarse", es decir, mediante colisiones inelásticas con electrones libres de cierta energía, subir alguno de los electrones que contiene a niveles de energía superior. Posteriormente, cuando estos electrones se "desexcitan", pierden la energía adquirida y la emiten en forma de radiación electromagnética ultravioleta. Ello se consigue, normalmente, haciendo pasar una corriente por el tubo que proporcione la energía suficiente para conseguir la ionización del gas noble que acompaña al mercurio y mantenerla. Sin embargo, al comienzo del encendido, el vapor de mercurio y el gas argón se comportan como un aislante y, por tanto, no conducen la corriente eléctrica. Para conseguir que circule una corriente por el tubo, debemos ionizar inicialmente el argón por otros métodos; de ello se encargará una alta tensión, aplicada entre los extremos del tubo, en los que se encuentran situados sendos filamentos hechos de tungsteno, que emiten electrones cuando se calientan y sirven también de electrodos para establecer la alta tensión mencionada. (Ver la figura 1). 


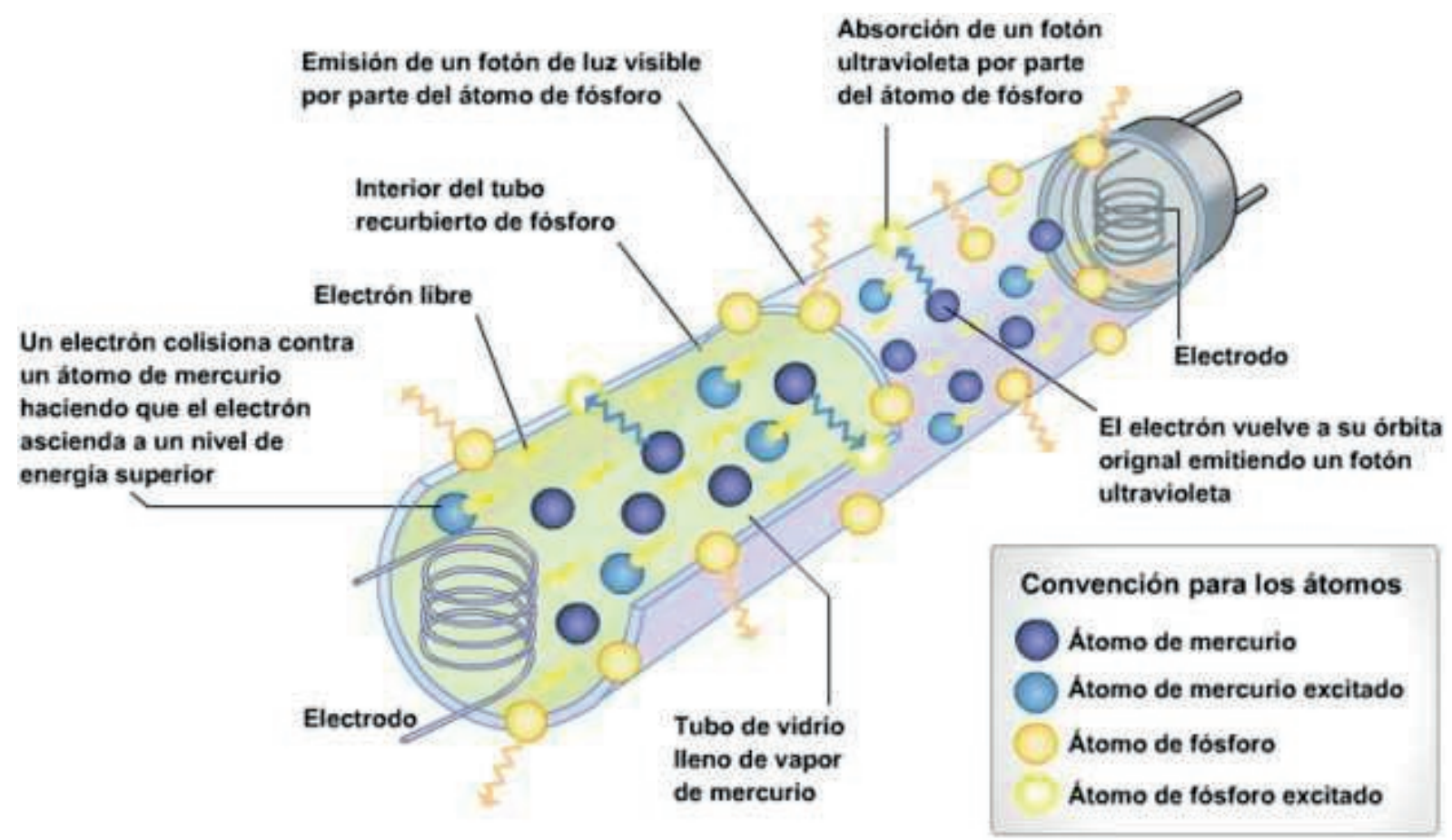

Figura 1: Esquema de los fenómenos que tienen lugar en el interior de un tubo fluorescente. Imagen tomada de Wikipedia

Si se utilizara sólo mercurio, también se podría obtener luz. En realidad, eso supondría construir una lámpara de mercurio como las empleadas en ciertos casos. No obstante, ello presenta muchos inconvenientes:

a) La cantidad de mercurio en la lámpara debería ser mayor;

b) Se obtendría luz con una fuerte componente visible, azulado verdosa, del plasma de mercurio, inadmisible para iluminación doméstica.

c) Se necesitarían tensiones de ionización inicial mucho mayores, próximas al arco voltaico, que las utilizadas para ionizar el argón;

d) Para mantener la ionización también se necesitarían tensiones mayores; 
e) La corriente de funcionamiento en el plasma sería excesiva, en detrimento de la vida útil del tubo;

f) La presión del vapor de mercurio alcanzada en el interior del tubo sería más pequeña y se debería usar un vidrio más grueso;

g) El precio sería excesivo para uso doméstico.

Tras la ionización, quedan átomos neutros de argón que también se "excitan" por las colisiones con los electrones libres del plasma formado. Al perder energía, los electrones excitados la reemiten en forma de radiación electromagnética visible no blanca y, además, con mucha menos intensidad que el revestimiento de fósforo. Por ello, el argón contribuye algo a la luminosidad del tubo, pero la iluminación que proporciona no es eficiente, razón por la cual se necesita el mercurio y el recubrimiento de fósforo de las paredes del tubo.

Como hemos apuntado más arriba, la luz emitida por un tubo fluorescente no puede provenir directamente del vapor de mercurio que llena el tubo a más o menos presión, pues esa luz es fundamentalmente ultravioleta, con una pequeña componente en el rango del azul. La radiación ultravioleta emitida por el mercurio es absorbida por el recubrimiento, en forma de polvo, de las paredes del tubo que, a su vez, la reemite en el espectro visible; su coloración depende del compuesto utilizado en dicho recubrimiento interno.

El tubo es de vidrio porque este material es transparente a la luz visible pero opaco a la radiación ultravioleta. De utilizar un material irrompible, dejaría pasar la radiación ultravioleta, lo cual haría inaceptable el uso de estas lámparas fluorescentes, debido al consiguiente riesgo para la salud. 
¿Cómo se consigue que el gas noble se ionice inicialmente, pasando al estado de plasma y permitiendo el paso de la corriente? De ello se encargan los electrones emitidos por los filamentos calientes (situados en los extremos del tubo), que son, a su vez, acelerados por la descarga de alta tensión producida por la reactancia.

Si las lámparas fluorescentes funcionaran en continuo, aumentaría progresivamente el grado de ionización del argón, reduciendo la resistencia del gas al paso de la corriente $y$, en consecuencia, aumentando la intensidad de ésta, lo que, a su vez, produciría más ionización, provocando una reacción en cadena que destruiría la lámpara en pocos segundos. Para evitarlo, se necesita, una vez establecida la descarga, primero anular la alta tensión y después intercalar un limitador de corriente, y de esa función se encargan, respectivamente, el cebador y la reactancia inductiva, constituida ésta por una bobina enrollada sobre un núcleo de chapas de acero. Estas chapas ferromagnéticas intensifican el campo magnético en el interior de la bobina y son las responsables del zumbido característico de los tubos fluorescentes, debido a las vibraciones que experimentan por estar sometidas a un campo magnético oscilante (ese mismo zumbido se detecta en los transformadores de tensión, por la misma razón).

Mientras el tubo esté encendido, una tensión baja y alterna (la de la red doméstica, por ejemplo) permite la existencia de una corriente pulsante que conserva la ionización del gas noble a niveles adecuados y proporciona la energía necesaria para establecer un equilibrio entre la ionización del argón y la recaptura de los electrones por éste, es decir, para mantener la descarga. Los electrones de esa corriente, en sus colisiones con los átomos neutros del vapor de mercurio, excitan los electrones de su interior. La bobina, por su parte, se opone a que la corriente aumente demasiado (ver los dos párrafos siguientes), manteniéndola entre los límites apropiados. La corriente pulsante del funcionamiento normal del tubo obliga a la luz emitida a parpadear a la misma frecuencia que oscila aquella. 
Recapitulando, necesitamos una descarga al comienzo del funcionamiento que no vuelva a aparecer mientras el tubo se mantenga encendido y una corriente de trabajo por debajo de un cierto límite. En nuestra ayuda viene la Ley de Faraday-Lenz.

Una bobina alimentada por una corriente produce un campo magnético que se mantiene en su interior y cuyo valor es función de la corriente y del número de espiras por unidad de longitud de la bobina. Si este campo magnético del interior de la bobina se anula porque la corriente que lo crea desaparece, se genera en la bobina una tensión que es proporcional al número de espiras de la bobina y al ritmo de anulación del campo magnético (B), es decir, a la rapidez con que desaparece la corriente que lo crea y el valor máximo del campo -de ahí las chapas metálicas que aumentan el valor de B- (ley de Faraday). Esa tensión es tal que induce una corriente en la bobina que se opone a que el campo magnético disminuya (ley de Lenz). De forma análoga, si la corriente aumenta, la inducción se opondrá a dicho aumento. De este modo la bobina puede mantener una intensidad de corriente entre ciertos límites, mientras el tubo está encendido, y provocar una alta tensión al inicio de la operación.

Veamos cómo se puede crear la alta tensión inicial.

De acuerdo con lo anterior, para establecer una alta tensión podemos hacer dos cosas, anular el campo magnético lentamente en una bobina muy grande (de muchas espiras) o anular el campo magnético muy rápidamente, para lo cual nos es suficiente una bobina de menor tamaño, más adecuada para su uso y, por supuesto, más barata. Esta alta tensión la conseguimos en un tubo fluorescente con una bobina relativamente pequeña gracias a un sistema adicional que anula el campo magnético inicial de forma muy rápida. 
La frecuencia de la tensión sinusoidal proporcionada por la red eléctrica $(50 \mathrm{~Hz})$ no es suficiente para anular el campo magnético de forma suficientemente rápida como para permitir la utilización de una bobina pequeña en la creación de una alta tensión. Además, existe otro importante factor que nos impide usar esa tensión sinusoidal. Si la bobina fuera lo suficientemente grande como para crear la alta tensión necesaria para iniciar la descarga con la tensión alterna de la red, esta alta tensión estaría generando descargas a la frecuencia de alimentación, provocando un efecto de avalancha en la ionización y la consiguiente destrucción del tubo. La bobina es imprescindible para mantener la corriente de trabajo entre ciertos límites, por lo que no puede ser desconectada una vez iniciada la descarga; si se hiciera así, serían necesarias dos bobinas, una para iniciar y otra para mantener encendido el tubo. El cebador se encarga de conseguir ambas cosas con una única bobina (ver la figura 2).

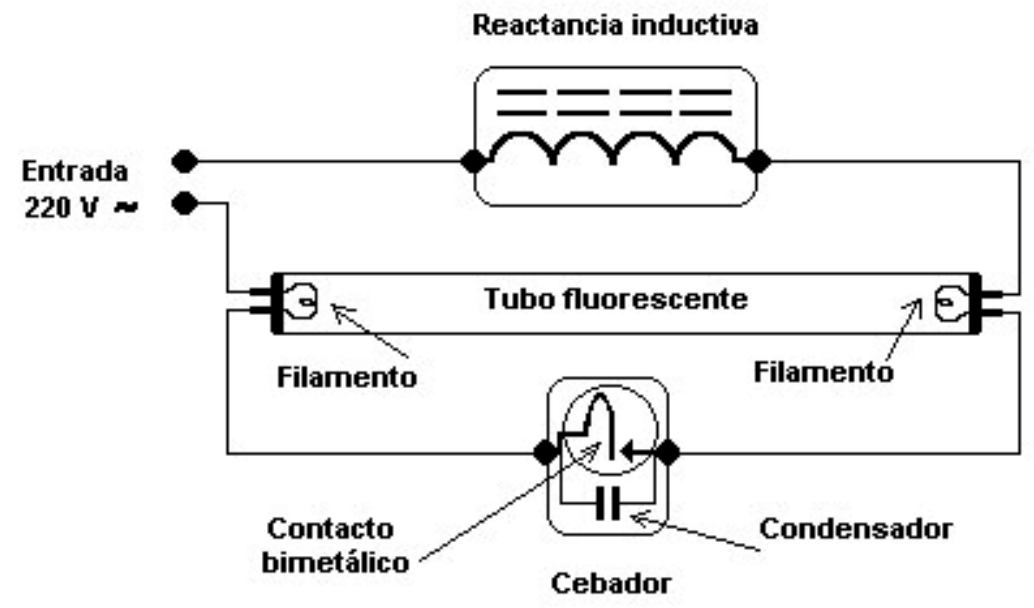

Figura 2: Esquema eléctrico del conjunto de una lámpara fluorescente. Imagen tomada de Wikipedia

El cebador, también llamado partidor, es una pequeña ampolla de cristal que contiene un gas noble a baja presión y un contacto formado por dos láminas metálicas. El estado normal de este contacto es "abierto", de forma que no permite el 
paso de la corriente. La bobina y los electrodos permanecen entonces desconectados aunque esté conectada la alimentación. Sin embargo, cuando se conecta la alimentación, entre las láminas separadas se establece un campo eléctrico de suficiente intensidad como para ionizar el gas noble de la ampolla, aumentando su temperatura. Las láminas entonces se deforman por el calor y se juntan, cerrando el circuito y permitiendo el paso de la corriente por los filamentos y la bobina, estableciendo en ésta el campo magnético y haciendo que aquellos emitan electrones en los extremos del tubo para iniciar la ionización y reducir la resistencia del gas del tubo al paso de la corriente.

A continuación, al cerrase el contacto, el gas noble de la ampolla del cebador se recombina con los electrones y disminuye su temperatura, con lo que se reduce la deformación de las láminas y se vuelve a abrir el circuito. Esta apertura trae como consecuencia el apagado de los electrodos y que el campo magnético creado en la reactancia inductiva se anule de forma muy rápida y, de acuerdo con la ley de inducción de Faraday-Lenz, se genera el alto voltaje entre los filamentos, que ahora actúan como electrodos, capaz de producir la descarga en el gas contenido en el tubo fluorescente, ionizando el argón, bajando drásticamente la resistencia en el mismo y permitiendo el paso de la corriente a su través. La corriente fluye ahora por el interior del tubo y no deja energía suficiente para volver a ionizar el gas del cebador, con lo que éste permanece desconectado en lo sucesivo, hasta el inicio de una nueva operación. Por esta razón, si, una vez encendido el sistema, se retira el cebador, el tubo sigue funcionando.

De hecho, un cebador se suele estropear porque sus láminas se quedan pegadas, por lo que no se puede generar la alta tensión y los filamentos permanecen encendidos emitiendo electrones. Estos electrones emitidos, aunque ionicen el gas, no son suficientes para provocar la descarga, además la corriente fluye sin dificultad por los contactos pegados del cebador, es decir, con resistencia nula. Se puede ver entonces 
la luminosidad en los extremos del tubo provocada por la incandescencia de los filamentos. En tales casos, es una práctica habitual, para conseguir el encendido del tubo, desconectar el cebador, por el simple método de retirarlo, lo que provoca la alta tensión en la bobina y el apagado de los filamentos.

En paralelo con el contacto bilaminar del cebador se instala un condensador, destinado, simplemente, a evitar las posibles chispas que podrían aparecer entre las láminas del contacto cuando se abren, debido a la alta tensión generada por la bobina. Este condensador no es imprescindible para el funcionamiento del tubo fluorescente, pero sí ayuda a aumentar la vida útil del contacto bilaminar, salvando al contacto de quedarse "pegado". Ésta es una de las razones por las que se recomienda usar la iluminación fluorescente en regímenes continuos, evitando el apagado y el encendido del sistema con frecuencia.

El otro componente que puede fallar por el encendido y apagado frecuente es la bobina, como consecuencia de las grandes corrientes que llegan a establecerse, por causa de la inducción, en el inicio de la operación de encendido. No obstante, el cebador suele ser la primera pieza del conjunto en estropearse; los gases y el revestimiento de fósforo suelen durar mucho más.

Cuando el cebador funciona normalmente pero no consigue iniciar la descarga, está en condiciones de empezar de nuevo y lo seguirá intentado una y otra vez. Ello puede ocurrir porque la bobina esté estropeada, y no dé la suficiente tensión para iniciar la descarga, o porque el tubo esté "gastado", es decir, el gas no tenga las condiciones para su ionización. En tales casos el tubo parpadea ostensiblemente, sin llegar a encenderse, y se pueden oír claramente los chasquidos de los contactos del cebador al abrirse y cerrarse. 
Cuando una lámpara fluorescente tarda en encenderse o al hacerlo parpadea, se eleva el consumo de electricidad y se acelera el desgaste de la propia lámpara. Éste es un síntoma de que el sistema se encuentra en mal estado. Otro de los síntomas es el ennegrecimiento de los extremos del tubo, señal de que los filamentos se han desgastado.

Obviamente, en una bombilla de bajo consumo moderna se han sustituido la reactancia y el cebador por la electrónica correspondiente a fin de producir el mismo efecto. Además, se consigue convertir la frecuencia de $50 \mathrm{~Hz}$ de la corriente en el tubo hasta mucho más altas frecuencias, del orden de 35.000 a 40.000 Hz. Estas altas frecuencias aseguran una luz casi estable, sin los parpadeos a $50 \mathrm{~Hz}$ de las lámparas fluorescentes normales, proporcionando un ambiente más confortable y evitando el efecto estroboscópico que prohibía le uso de fluorescentes para iluminar maquinas en movimiento pulsante o rotatorio. Dicho efecto hacía que, si la máquina oscilaba o rotaba a una frecuencia múltiplo de $50 \mathrm{~Hz}$, el usuario la viera parada, con el consiguiente peligro de uso.

Hagamos un resumen:

1) En perfectas condiciones de funcionamiento, cuando alimentamos una lámpara fluorescente, conectándola a la red doméstica de tensión alterna, la corriente no puede fluir por ninguno de los elementos del sistema porque el cebador está abierto y el gas del tubo se comporta como un aislante.

2) Sin embargo, entre las láminas del cebador se establece la tensión de la red y, como están muy próximas, el campo eléctrico entre ellas es lo suficientemente grande como para provocar la ionización del gas noble contenido en la ampolla. Ello aumenta la temperatura y hace que las láminas que constituyen los electrodos del cebador se curven y se junten cerrando el circuito. 
3) Con el contacto del cebador cerrado, se permite el paso de la corriente por los filamentos del tubo que se tornan incandescentes y emiten los electrones que inician la ionización del gas noble que acompaña al vapor de mercurio, creando las condiciones previas para que la alta tensión posterior inicie la descarga y pueda establecerse la corriente por el gas del tubo. Simultáneamente, la corriente pasa por la bobina, estableciendo el campo magnético en su interior.

4) Por estar los electrodos del cebador unidos, desaparece el campo eléctrico que ionizaba el gas de la ampolla y, en consecuencia, disminuye la temperatura del mismo, con lo que la deformación de las láminas del contacto desaparece y el circuito se abre.

5) La interrupción de la corriente, además de apagar la incandescencia de los filamentos del tubo, provoca la anulación muy rápida del campo magnético establecido previamente en la bobina, induciendo un alto voltaje en ésta, que se aplica a los filamentos, ahora funcionando como electrodos.

6) Evidentemente, la misma tensión se aplica entre los contactos del cebador. Sin embargo, mientras el gas del tubo ya está parcialmente ionizado, gracias a la emisión de los electrones por el caldeo de los filamentos, $\mathrm{y}$, por tanto, presenta una resistencia relativamente baja al paso de la corriente, el gas contenido en el cebador necesita ionizarse de nuevo. Consecuentemente, la respuesta del gas del tubo es mucho más rápida y la descarga se realiza en su seno, provocando una ionización mayor, es decir, el establecimiento de un plasma, un descenso grande de su resistencia y permitiendo a la corriente fluir a su través sin apenas dificultad, valiéndose de los dos electrodos.

7) El cebador ya no dispone de energía para ionizar el gas de su ampolla y se mantiene desconectado en lo sucesivo. 
8) La corriente del tubo está alimentada ahora por la tensión de la red y pulsa a una frecuencia de $50 \mathrm{~Hz}$. Pero como la bobina esta intercalada en el circuito, por ella pasa esa misma corriente, y, en respuesta a la ley de Faraday-Lenz, se opone a la pulsación y mantiene la tensión entre electrodos en los límites convenientes para evitar un efecto avalancha en la ionización del gas argón y su consecuente destrucción.

9) Mientras la corriente, que es relativamente elevada, se mantenga entre los límites adecuados, la ionización del gas del tubo se mantendrá en equilibrio. Dicha corriente provoca que los electrones libres del plasma colisionen con los átomos neutros del vapor del mercurio y del gas noble, aumentando la ionización de este último. Pero como no es excesivamente elevada, permite que algunos electrones sean recapturados por los iones. En definitiva, el equilibrio se mantiene.

10) Los electrones libres que se mueven formando parte de la corriente (la otra parte corresponde al movimiento más lento de los iones en sentido contrario), colisionan con los átomos neutros del vapor de mercurio. Estas colisiones ceden energía cinética a los electrones que permanecen orbitando en el átomo de mercurio y los "excitan" a órbitas más energéticas. Cuando la energía adquirida se pierde, porque los electrones "excitados" retornan a su órbita natural, se cede al medio en forma de ondas electromagnéticas de frecuencia ultravioleta.

11) Los fotones ultravioleta inciden sobre el recubrimiento de la pared interior del tubo, "excitando", a su vez, los electrones de los átomos de fósforo a energías superiores. Cuando estos electrones vuelven a su órbita natural, ceden la energía adquirida en forma de radiación electromagnética visible. De este modo se ilumina el tubo. 
12) Los electrones libres del plasma por el que circula la corriente también colisionan con los átomos neutros del gas noble que llena el tubo. Estas colisiones proceden de forma análoga a como lo hacen en el caso del mercurio. Cuando la energía adquirida por los electrones internos del gas noble se cede al medio, porque los electrones "excitados" retornan a su órbita natural, lo hace en forma de luz visible, pero esa luz, dada la presión del gas, es de poca intensidad y, además, dada la naturaleza del gas, no presenta las características de la luz blanca, necesaria para la iluminación doméstica.

\section{Ventajas no tan ventajosas}

La principal ventaja de una BFC frente a una bombilla de incandescencia es su eficiencia energética. Aproximadamente, se calcula un ahorro de un 25\% para un nivel de iluminación similar que no idéntico, pues el espectro de la luz de tungsteno es más uniforme en el rango visible que el correspondiente al de la luz fluorescente. $\mathrm{Su}$ temperatura de color habitual varía entre los $3.000 \mathrm{~K}$ y los $6.500 \mathrm{~K}$ (del Blanco Cálido a Luz Día Frío). No obstante, hoy se fabrican modelos con temperaturas de color más aproximadas a la iluminación incandescente, que van desde los $2.700 \mathrm{~K}$ hasta los $8.000 \mathrm{~K}$

Otra ventaja, nada despreciable es su vida útil, entre 5 y 15 veces mayor que la correspondiente a una bombilla de incandescencia (de 5.000 a 15.000 horas de funcionamiento), aunque ello depende de muchos factores, tales como el tipo de lámpara fluorescente, el equipamiento complementario utilizado $\mathrm{y}$, sobre todo, del número de veces que se apague y se encienda. En realidad, su vida útil se debería medir en función del número de encendidos. Por ejemplo, una lámpara utilizada 8 horas diarias ininterrumpidas puede llegar a tener una vida útil de 3.000 horas, mientras que la misma lámpara utilizada 16 horas diarias puede alarga su vida al 
doble del valor anterior. En resumen, nunca podemos fiarnos de los datos dados por el fabricante en cuanto a la duración de una BFC.

El ahorro en energía puede estar completamente compensado, incluso superado, por la necesidad de mantener encendida la bombilla a fin de paliar su elevado coste con el aumento de su duración. Si se quiere que una BFC dure lo especificado por el fabricante, se deberán mantener todas las bombillas de una casa habitualmente encendidas. Por otra parte, pese que las BFC ya no tardan mucho en encenderse, como lo hacen los tubos fluorescentes clásicos, su luminosidad máxima tarda un tiempo bastante largo en conseguirse, como comentamos en el epígrafe siguiente.

\section{Desventajas}

Las lámparas fluorescentes tienen un rendimiento luminoso que se puede estimar entre 50 y 90 lúmenes por vatio $(\mathrm{lm} / \mathrm{W})$ de potencia. Sin embargo, dado su funcionamiento, la luminosidad de la lámpara depende fundamentalmente de la superficie emisora, es decir de la superficie del cilindro iluminado, es por ello que una lámpara fluorescente clásica moderna de $18 \mathrm{~W}$ mide $60 \mathrm{~cm}$; la de $36 \mathrm{~W}$, 1,20 m y la de $54 \mathrm{~W}, 1,80 \mathrm{~m}$. Por esta misma razón, las $\mathrm{BFC}$ de más de $22 \mathrm{~W}$ no están disponibles en los puntos de venta habituales, pues su tamaño las hace inaceptables para uso doméstico.

El conjunto fluorescente tiene elementos reactivos (bobina y condensadores) que consumen potencia, pero también la devuelven a la red, lo cual es inadmisible, por el aumento de consumo para el usuario y por la propia compañía, pues esa potencia reactiva es indeseada en la red. Para evitarlo, se debe intercalar entre los terminales de entrada un condensador que tiene la finalidad de compensar la potencia reactiva, haciendo que toda la potencia tomada de la red se consuma en el conjunto. Por esta razón, a este tipo de arreglo se le denomina compensación en paralelo. Un pequeño 
cálculo permite saber el valor del condensador a intercalar (puede consultarse en Wikipedia).

Las lámparas fluorescentes no dan una luz continua, muestran un parpadeo correspondiente a la frecuencia de la corriente eléctrica aplicada. Aunque no se nota mucho a simple vista, una exposición continua a esta luz puede causar dolor de cabeza. El efecto es el mismo que si se configura una pantalla de ordenador a $50 \mathrm{~Hz}$.

Este parpadeo puede, además, causar un efecto estroboscópico, de forma que un objeto que gire u oscile a una velocidad múltiplo a la frecuencia de parpadeo puede verse estático bajo una luz fluorescente. Por ello, en algunos lugares (como talleres con maquinaria) no es recomendable el uso de luz fluorescente. También puede causar problemas con las cámaras de vídeo, ya que la frecuencia a la que el sensor lee la imagen puede coincidir con las fluctuaciones en intensidad de la lámpara fluorescente.

Las lámparas fluorescentes no pueden conectarse a un atenuador normal o dimmer de intensidad luminosa (un regulador para controlar el brillo). Sin embargo, hoy ya existen lámparas (de 4 contactos) y controladores especiales que permiten usar un interruptor con regulador de intensidad.

Como hemos mencionado más arriba, desde mediados de la década de los 80 del pasado siglo, hay una solución electrónica que evita algunos de estos inconvenientes. Esta electrónica hace funcionar al tubo de la misma manera que en la forma tradicional, pero a una frecuencia de 35.000 a $40.000 \mathrm{~Hz}$, con lo que se aumenta en un $10 \%$ el rendimiento, se evita mucho el efecto estroboscópico y el parpadeo se hace prácticamente indetectable por el ojo humano y las cámaras de vídeo. 
También hemos mencionado el hecho de que las lámparas fluorescentes necesitan unos minutos de calentamiento antes de alcanzar su flujo luminoso normal, por lo que es aconsejable utilizarlas en lugares donde no se están encendiendo y apagando continuamente (como pasillos y escaleras). Por otro lado, los encendidos y apagados constantes acortan notablemente su vida útil. Los accesorios electrónicos, que sustituyen a la reactancia y al cebador tradicionales, hacen instantáneo el encendido del tubo, alargando de esta manera la vida útil, pero, no obstante, una BFC siempre tarda bastante tiempo en llegar a su luminosidad normal.

En un fluorescente clásico, el usuario puede sustituir el tubo, el cebador o la bobina de forma independiente. En una BFC, si cualquiera de los elementos se estropea, la bombilla completa debe desecharse.

El peor inconveniente de la iluminación fluorescente es que un solo tubo contiene suficiente mercurio como para contaminar 30.000 litros de agua. 